\title{
Materials selection in micro- or nano-mechanical design: Towards new Ashby plots for small-sized materials
}

\author{
Yu Zou ${ }^{\mathrm{a}, \mathrm{b}}$ \\ ${ }^{a}$ Department of Materials, ETH Zurich, Vladimir-Prelog-Weg 5, CH-8093 Zurich, Switzerland \\ ${ }^{b}$ Department of Mechanical Engineering, Massachusetts Institute of Technology, 77 \\ Massachusetts Avenue, Cambridge, Massachusetts 02139, USA
}

\begin{abstract}
Conventional Ashby plots for material selection may not appropriately describe material properties at small-length scales, new plots taking account of size effects are urgently needed for micro- or nano-mechanical design. Here, I show strength-modulus, strength-density, and size effect-Peierls stress plots for various solid materials at small scales.
\end{abstract}

Keywords: Size effect; Plastic deformation; Micro-compression; Focused ion beam; Small scales; Ashby plots

*Correspondence should be addressed to Y.Z. (Department of Mechanical Engineering, Massachusetts Institute of Technology, 77 Massachusetts Avenue, Cambridge, Massachusetts 02139, USA. Email: yuzou@mit.edu) 


\section{Introduction}

For decades, Ashby plots for material selection have provided a qualitative guide for choosing appropriate materials for engineering applications [1], such as strength-density and strengthmodulus charts [2]. The conventional Ashby plots were established based on the properties of coarse-grained or single-crystalline materials in their bulk forms. At micrometer or nanometer length scales, the material properties, however, may not be identical to those of the bulk ones. Size dependence of strength in metals presents a good example of this multiscale complexity [3]: at the nanometer scale $(<\sim 100 \mathrm{~nm})$, the activities of an individual dislocation and material surfaces may dominate the material strength; at sub-micrometer scales $(\sim 100 \mathrm{~nm}-1 \mu \mathrm{m})-\mathrm{a}$ length scale of mean dislocation spacing, dislocation segments may control the strength; at micrometer scales $(\sim 1 \mu \mathrm{m}-100 \mu \mathrm{m})$, one may observe a transition between small-scale strength to macroscopic strength, where the latter represents the strength used in conventional Ashby plots. Consequently, conventional Ashby plots may not appropriately describe material properties when the length scale of a material is reduced into micrometer and nanometer regimes.

Micro- or nanoelectromechanical systems (MEMS or NEMS) generally consist of many components in the dimension from a few micrometers to a few nanometers. Appropriate selection of materials with desired properties is critical to overall functionality and reliability of the systems. Therefore, to create new Ashby-type plots that take account of the size dependence of material properties is highly desirable to meet the challenge of rapid development of microelectronics and nanotechnology sectors. 
In 2004, Uchic and his co-workers first reported size-dependent strength of micrometer-sized metal pillars, in which Ni micro-pillars were fabricated using the focused ion beam (FIB) milling technique and compressed by a nanoindenter with a flat-punch tip [4]. Over a decade, significant advances have been made in applying this methodology to study mechanical properties of materials in small dimensions - usually between a few microns down to 100 nanometers. A large number of materials reveal strong size dependence of strength when their sample dimensions are reduced into the micro- and nanoscales. Meanwhile, the yield strengths of these micro/nanopillars can attain a significant fraction of their theoretical ones. Dou and Derby [5] quantify the micro-compression data using a power-law fit, as:

$$
\frac{\sigma}{G}=\mathrm{A}\left(\frac{D}{b}\right)^{-m}
$$

where $\sigma$ is yield strength or flow stress, $G$ is the shear modulus of a corresponding slip system, $A$ is a constant, $D$ is the pillar diameter, $b$ is the Burgers vector, and $m$ is so-called size-effect exponent. Since the Uchic's work was published, over a thousand related studies have been reported, which covers a broad range of materials (see reviews [6-8]). Table 1 presents a summary of some representative materials: pure metals (e.g., face-centered-cubic (fcc) $\mathrm{Au}[9$, 10], $\mathrm{Al}[11,12], \mathrm{Ni}[13]$, and $\mathrm{Cu}[14,15]$; body-centered-cubic (bcc) Nb, Ta, Mo, and W [16, 17]; and hexagonal close packed (hcp) $\mathrm{Ti}[18,19]$ and $\mathrm{Mg}$ [20]), ionic crystals (e.g., $\mathrm{NaCl}$ [21], $\mathrm{KCl}$ [22], $\mathrm{LiF}[23,24]$, and $\mathrm{MgO}$ [25]), covalent crystals (e.g., Si [26] and GaAs [27]), ordered intermetallics (e.g., TiAl [28]), disordered intermetallics (e.g., high-entropy alloys (HEAs) [29, 30]), complex metallic alloys (CMAs, e.g., $\mathrm{Al}_{13} \mathrm{Co}_{4}[31]$ ), quasicrystals (QCs, e.g., icosahedral Al-Pd-Mn [32] and decagonal Al-Ni-Co [33]) and metallic glasses (MGs, e.g., Cu- and Zn-based ones [34]). The measured $m$ values in fcc metals all fall in the range of $\sim 0.6-0.7$. Such value is 
nearly identical to all FIB-milled fcc micro-pillars. Later investigations reveal that bcc metal pillars (Nb, Mo, Ta, W [16, 17, 35] and V [36]) have a large range of size effect exponents ( $m$ of $\sim 0.2-0.9$ ) and hcp metal pillars exhibit an exponent depending on slip systems ( $m$ of $\sim 0.4$ for prismatic slip; $m$ of $\sim 0.6$ for basal slip[8]). When materials dimensions are reduced into the submicrometer-sized regime, even hard and brittle materials exhibit plasticity to a certain extent, such as Si [26], quasicrystals [32], and metallic glasses [37]. They are all found to show minor or almost no size effect. For example, in the sample size range from $\sim 100 \mathrm{~nm}$ to $\sim 1 \mu \mathrm{m} \mathrm{[38],} \mathrm{Si}$ exhibits much smaller size effects than most metals.

One may ask what the Ashby plots look like after taking account of such size effects and whether there is a general law describing the size effects in all these inorganic solids. Here, this work presents material-property plots for micro/nano-pillars, compare the differences among them, and unveils a general trend of the size effects.

\section{Results and discussion}

This work focuses on the literature of micro/nano-pillars produced by $\mathrm{Ga}^{+}-\mathrm{FIB}$ milling, because a vast majority (over 90\%) of reported micro-compression results are based on a $\mathrm{Ga}^{+}$-FIB system, which is commonly and commercially available. Although a few well-known issues are found to be associated with the FIB-milling process, such as FIB-induced irradiation damage and taper angle [39]. Nevertheless, the FIB technique has so far been the most important and widely used method to prepare small-sized samples for mechanical measurement. In addition, the Ashby-type plots for small-sized materials are simplified in two aspects: Because $m$ also depends on initial defect density of a sample [40, 41], only well-annealed samples are compared here; For the 
reason of legibility, the highest strengths of micro/nano-pillars are compared here, which generally correspond to a pillar diameter of $\sim 100-200 \mathrm{~nm}$.

The highest strength of a micro/nano-pillar sample as a function of its shear modulus, $G$, is plotted in Figure 1. The critical resolved shear stresses (CRSS) of all the materials fall in the range between $\sim G / 30$ and $\sim G / 100$. It is interesting to note that the CRSS of some covalent crystals ([26, 27]), quasicrystals [32, 33], and metallic glasses [34] can approach their theoretical values $(\sim G / 10[42])$. As a contrast, most metals, such as $\mathrm{Mo}, \mathrm{W}, \mathrm{Cu}, \mathrm{Al}$ and $\mathrm{Mg}$, are near to the lower strength boundary $(\sim G / 100)$. The difference of strengths might be attributed to relatively low grown-in defect density or FIB-induced damage levels in the former group.

Taking into account of size dependence of material strength, traditional strength-modulus plots that are developed for bulk materials may not be valid anymore, and, therefore, the new plots that include size effect have to be created. Two examples are illustrated here in Figure 2 and Figure 3, showing the relations of CRSS vs. Young's modulus and CRSS vs. density, respectively. For instance, if Young's modulus and yield strength are the only determinant parameters, MgO, NbTaMoW HEAs, TiAl, quasicrystals, and Si should be good candidate materials for structural applications. For another example, when specific strength (i.e., strengthto-density ratio) is the most critical factor to be considered, $\mathrm{MgO}, \mathrm{Si}, \mathrm{TiAl}$, and quasicrystals are good candidates, as shown in Figure 3. Here, Si micro/nano-pillars offer the highest specific strength among all the micro/nano-pillars reported to date, indicating micro- or nanoscale $\mathrm{Si}$ is able to store elastic energy as high as $\sim 1 \mathrm{MJ} / \mathrm{kg}$, which is comparable to that of lithium battery. However, in real engineering applications, many other important, sometimes even more 
dominating, factors should be considered, such as creep resistance, fracture toughness, electrical conductivity, thermal conductivity, etc. Unfortunately, only few of these properties are completely known for materials at such small dimensions.

The other question concerns how one can predict the size effect for a material only based on its known characteristics. It is found, in general, that brittle materials exhibit a minor or no size effect, such as Si [26], metallic glasses [34], and quasicrystals [32, 33], while ductile materials show relatively large size effect, such as fcc metals, basal-slip hcp metals, and ionic crystals on soft slips. It is known that the brittleness of a material can be qualitatively correlated to its energy barrier or force that resists dislocation motion (or shear band propagation for metallic glasses). This force can be described by the Peierls stress, $\tau_{\mathrm{p}}$ (or shear stress at $0 \mathrm{~K}$ for metallic glasses). Figure 4 illustrates $m$ as a function of $\tau_{\mathrm{p}}$ for different types of materials in a linear- logarithmic format. With the value of $m$ ranging from 0 to about 0.8 , and $\tau_{\mathrm{p}}$ covers over nearly three orders of magnitude from $\sim 0.001 \mathrm{GPa}$ to $\sim 1 \mathrm{GPa}$. A general trend of the relation between the Peierls stress and size effect is found: when $\tau_{\mathrm{p}}<\sim 0.1 \mathrm{GPa}, m \approx 0.6-0.8$, showing a relatively consistent value; when $\sim 0.1 \mathrm{GPa}<\tau_{\mathrm{p}}<\sim 1 \mathrm{GPa}, 0<m<\sim 0.6-0.8$, showing a decreased $m$ with increasing $\tau_{\mathrm{p}}$; when $\tau_{\mathrm{p}}>\sim 1 \mathrm{GPa}, m \approx 0$, showing almost no size effect. This observed trend can be associated with the ratio between the required stress for dislocation nucleation and the stress for dislocation motion (or the stress for shear band formation to that for shear band propagation for metallic glasses). For example, in a 100-nm Ni pillar, more than $95 \%$ of the flow stress is contributed by dislocation source; while in a 100-nm W pillar, less than 50\% of the flow stress is due to source strengthening, figuratively speaking. It should be noted that Figure 4 represents the size effects of materials that are only measured at room temperature and nearly identical strain rate $\left(\sim 10^{-3} \mathrm{~s}^{-1}\right)$ 
and the size dependence of a material strength may depend on the testing temperature and strain rate. Some previous studies demonstrate three-dimensional (3D) size-temperature-strength deformation maps, such as in Si [38] and quasicrystals [43], but the systematic studies on various materials over a large temperature and strain rate range are still scarce. Nevertheless, although the detailed underlying strengthening mechanism may vary among different materials, this general trend in Figure 4 can be technologically important, providing a common guide to predict material strengths at multi-scales.

\section{Summary and outlook}

In summary, the material-selection plots for micrometer- and submicrometer-sized materials have been created. The strength-modulus plot shows that the highest strengths in various FIBmilled pillars fall in the range of $\sim G / 30-G / 100$. The strength-to-density plot compares specific strength of various materials at small dimensions, indicating that $\mathrm{Si}$ possesses the highest strength-to-density ratio reported to date. The size effect-Peierls stress log-log plot demonstrates three size-effect regimes ( $m \approx 0.6-0.8,0<m<\sim 0.6-0.8$ and $m \approx 0)$ with increasing resistance for the propagation of plasticity mediums - dislocations or shear bands.

The superior and useful mechanical properties of the small-scale materials presented here may permit their implementation in critical applications as well as offer a strong motivation for their further development. For future studies, it is important to collect data and create Ashby-type plots for small-sized materials without FIB effects as well as including other material properties, such as thermal and electrical properties. It is also instrumental to create 3D Ashby-type mechanism maps that takes account of sample size, temperature, and strain rate, in which several 
deformation mechanisms may appear, such as dislocation glide, diffusional flow, and dislocation creep. 


\section{Acknowledgements}

The author thanks Professor Ralph Spolenak (ETH Zurich) for careful review of the manuscript and helpful discussions and Dr. A. Evans for proofreading. The author also acknowledges the financial supports through the Swiss National Science Foundation (SNF project grant: 200021_143633, and SNF Early Postdoc.Mobility: P2EZP2_165278).

\section{References}

[1] M.F. Ashby, D. Cebon, Materials selection in mechanical design, Le Journal de Physique IV 3(C7) (1993) C7-1C7-9.

[2] M.F. Ashby, Chapter 4 - Material Property Charts, Materials Selection in Mechanical Design (Fourth Edition), Butterworth-Heinemann, Oxford, 2011, pp. 57-96.

[3] M. Zaiser, A. Seeger, Chapter 56 Long-range internal stresses, dislocation patterning and work-hardening in crystal plasticity, in: F.R.N. Nabarro, M.S. Duesbery (Eds.), Dislocations in Solids, Elsevier2002, pp. 1-100.

[4] M.D. Uchic, D.M. Dimiduk, J.N. Florando, W.D. Nix, Sample dimensions influence strength and crystal plasticity, Science 305(5686) (2004) 986-989.

[5] R. Dou, B. Derby, A universal scaling law for the strength of metal micropillars and nanowires, Scripta Materialia 61(5) (2009) 524-527.

[6] M.D. Uchic, P.A. Shade, D.M. Dimiduk, Plasticity of Micrometer-Scale Single Crystals in Compression, Annu Rev Mater Res 39 (2009) 361-386.

[7] O. Kraft, P.A. Gruber, R. Monig, D. Weygand, Plasticity in Confined Dimensions, Annual Review of Materials Research, Vol 4040 (2010) 293-317.

[8] J.R. Greer, J.T.M. De Hosson, Plasticity in small-sized metallic systems: Intrinsic versus extrinsic size effect, Progress in Materials Science 56(6) (2011) 654-724.

[9] J.R. Greer, W.C. Oliver, W.D. Nix, Size dependence of mechanical properties of gold at the micron scale in the absence of strain gradients, Acta Mater 53(6) (2005) 1821-1830.

[10] C.A. Volkert, E.T. Lilleodden, Size effects in the deformation of sub-micron Au columns, Philos Mag 86(33-35) (2006) 5567-5579.

[11] A. Kunz, S. Pathak, J.R. Greer, Size effects in Al nanopillars: Single crystalline vs. bicrystalline, Acta Mater 59(11) (2011) 4416-4424.

[12] K.S. Ng, A.H.W. Ngan, Stochastic nature of plasticity of aluminum micro-pillars, Acta Mater 56(8) (2008) 1712-1720.

[13] C.P. Frick, B.G. Clark, S. Orso, A.S. Schneider, E. Arzt, Size effect on strength and strain hardening of smallscale [111] nickel compression pillars, Materials Science and Engineering: A 489(1-2) (2008) 319-329.

[14] D. Kiener, C. Motz, T. Schoberl, M. Jenko, G. Dehm, Determination of mechanical properties of copper at the micron scale, Advanced Engineering Materials 8(11) (2006) 1119-1125.

[15] A.T. Jennings, M.J. Burek, J.R. Greer, Microstructure versus Size: Mechanical Properties of Electroplated Single Crystalline Cu Nanopillars, Phys Rev Lett 104(13) (2010) 135503.

[16] A.S. Schneider, D. Kaufmann, B.G. Clark, C.P. Frick, P.A. Gruber, R. Monig, O. Kraft, E. Arzt, Correlation between Critical Temperature and Strength of Small-Scale bcc Pillars, Phys Rev Lett 103(10) (2009).

[17] J.-Y. Kim, D. Jang, J.R. Greer, Tensile and compressive behavior of tungsten, molybdenum, tantalum and niobium at the nanoscale, Acta Mater 58(7) (2010) 2355-2363.

[18] J. Sun, Q. Yu, Z.W. Shan, J. Li, X.X. Huang, L. Xiao, E. Ma, Strong crystal size effect on deformation twinning, Nature 463(7279) (2010) 335-338.

[19] Q. Sun, Q. Guo, X. Yao, L. Xiao, J.R. Greer, J. Sun, Size effects in strength and plasticity of single-crystalline titanium micropillars with prismatic slip orientation, Scripta Materialia 65(6) (2011) 473-476.

[20] E. Lilleodden, Microcompression study of Mg (0001) single crystal, Scripta Materialia 62(8) (2010) 532-535.

[21] Y. Zou, R. Spolenak, Size-dependent plasticity in micron- and submicron-sized ionic crystals, Philosophical Magazine Letters 93(7) (2013) 431-438. 
[22] Y. Zou, R. Spolenak, Size-dependent plasticity in $\mathrm{KCl}$ and LiF single crystals: influence of orientation, temperature, pre-straining and doping, Philos Mag 95(16-18) (2015) 1795-1813.

[23] E.M. Nadgorny, D.M. Dimiduk, M.D. Uchic, Size effects in LiF micron-scale single crystals of low dislocation density, J Mater Res 23(11) (2008) 2829-2835.

[24] R. Soler, J.M. Molina-Aldareguia, J. Segurado, J. Llorca, R.I. Merino, V.M. Orera, Micropillar compression of LiF 111 single crystals: Effect of size, ion irradiation and misorientation, Int J Plasticity 36 (2012) 50-63.

[25] S. Korte, W.J. Clegg, Discussion of the dependence of the effect of size on the yield stress in hard materials studied by microcompression of MgO, Philos Mag 91(7-9) (2011) 1150-1162.

[26] F. Ostlund, K. Rzepiejewska-Malyska, K. Leifer, L.M. Hale, Y.Y. Tang, R. Ballarini, W.W. Gerberich, J. Michler, Brittle-to-Ductile Transition in Uniaxial Compression of Silicon Pillars at Room Temperature, Adv Funct Mater 19(15) (2009) 2439-2444.

[27] F. Ostlund, P.R. Howie, R. Ghisleni, S. Korte, K. Leifer, W.J. Clegg, J. Michler, Ductile-brittle transition in micropillar compression of GaAs at room temperature, Philos Mag 91(7-9) (2011) 1190-1199.

[28] K. Edalati, S. Toh, H. Iwaoka, M. Watanabe, Z. Horita, D. Kashioka, K. Kishida, H. Inui, Ultrahigh strength and high plasticity in TiAl intermetallics with bimodal grain structure and nanotwins, Scripta Materialia 67(10) (2012) 814-817.

[29] Y. Zou, S. Maiti, W. Steurer, R. Spolenak, Size-dependent plasticity in an Nb25Mo25Ta25W25 refractory high-entropy alloy, Acta Mater 65(0) (2014) 85-97.

[30] A.M. Giwa, P.K. Liaw, K.A. Dahmen, J.R. Greer, Microstructure and small-scale size effects in plasticity of individual phases of Al0.7CoCrFeNi High Entropy alloy, Extreme Mechanics Letters.

[31] C. Walter, J.M. Wheeler, J.S. Barnard, R. Raghavan, S. Korte-Kerzel, P. Gille, J. Michler, W.J. Clegg, Anomalous yielding in the complex metallic alloy Al13Co4, Acta Mater 61(19) (2013) 7189-7196.

[32] Y. Zou, P. Kuczera, A. Sologubenko, T. Sumigawa, T. Kitamura, W. Steurer, R. Spolenak, Superior roomtemperature ductility of typically brittle quasicrystals at small sizes, Nat Commun 7 (2016).

[33] Y. Zou, P. Kuczera, W. Steurer, R. Spolenak, Disappearance of plastic anisotropy in decagonal quasicrystals at small scales and room temperature, Extreme Mechanics Letters (2016).

[34] C.Q. Chen, Y.T. Pei, J.T.M. De Hosson, Effects of size on the mechanical response of metallic glasses investigated through in situ TEM bending and compression experiments, Acta Mater 58(1) (2010) 189-200.

[35] S.M. Han, T. Bozorg-Grayeli, J.R. Groves, W.D. Nix, Size effects on strength and plasticity of vanadium nanopillars, Scripta Materialia 63(12) (2010) 1153-1156.

[36] R.M. D. Kaufmann, C.A. Volkert, O. Kraft,, Size dependent mechanical behaviour of tantalum, Int J Plasticity 27(3) (2011) 470-478.

[37] C. Volkert, A. Donohue, F. Spaepen, Effect of sample size on deformation in amorphous metals, J Appl Phys 103(8) (2008) 083539-083539-6.

[38] E. Hintsala, C. Teresi, W.W. Gerberich, Linking Nanoscales and Dislocation Shielding to the Ductile-Brittle Transition of Silicon, Metallurgical and Materials Transactions A (2016) 1-6.

[39] C. Volkert, A. Minor, Focused ion beam microscopy and micromachining, Mrs Bull 32(05) (2007) 389-399.

[40] J.A. El-Awady, M.D. Uchic, P.A. Shade, S.-L. Kim, S.I. Rao, D.M. Dimiduk, C. Woodward, Pre-straining effects on the power-law scaling of size-dependent strengthening in Ni single crystals, Scripta Materialia 68(3-4) (2013) 207-210.

[41] R. Gu, A.H.W. Ngan, Dislocation arrangement in small crystal volumes determines power-law size dependence of yield strength, J Mech Phys Solids 61(6) (2013) 1531-1542.

[42] T.H. Courtney, Mechanical behavior of materials, Waveland Press2005.

[43] Y. Zou, J.M. Wheeler, A.S. Sologubenko, J. Michler, W. Steurer, R. Spolenak, Bridging room-temperature and high-temperature plasticity in decagonal Al-Ni-Co quasicrystals by micro-thermomechanical testing, Philos Mag (2016) 1-23.

[44] P. Haasen, Dislocations and the Plasticity of Ionic-Crystals, Mater Sci Tech-Lond 1(12) (1985) 1013-1024.

[45] Y. Kamimura, K. Edagawa, S. Takeuchi, Experimental evaluation of the Peierls stresses in a variety of crystals and their relation to the crystal structure, Acta Mater 61(1) (2013) 294-309.

[46] S. Takeuchi, K. Edagawa, Chapter 8 Elastic and plastic properties of quasicrystals, in: F. Takeo, I. Yasushi (Eds.), Handbook of Metal Physics, Elsevier2008, pp. 267-311.

[47] W. Johnson, K. Samwer, A universal criterion for plastic yielding of metallic glasses with a (T/T g) $2 / 3$ temperature dependence, Phys Rev Lett 95(19) (2005) 195501.

[48] J.N. Wang, Prediction of peierls stresses for different crystals, Mat Sci Eng a-Struct 206(2) (1996) 259-269. 


\section{Figure captions}

Figure 1. The highest critical resolved shear stresses (CRSS) as a function of shear modulus, $G$, for representative micro-pillars in Table 1. It indicates the highest CRSS of various pillars is in the range of $\sim G / 30$ to $\sim G / 100$. Shear moduli are obtained from Refs. [44-47]. (For the reason of legibility, some references are not shown in the graph, but can be found in Table 1. the same reason for the other figures).

Figure 2. The highest CRSS as a function of Young's modulus, E, for representative micropillars, showing both high strength and Young's modulus for quasicrystals, high-entropy alloys, and $\mathrm{MgO}$ (Young's moduli are from Refs. [44-47]).

Figure 3. The CRSS as a function of density for representative micro-pillars, showing Si pillars are among the most high-strength and low-density pillars, while Au pillars have the lowest strength-to-density ratio (densities are calculated according to their compositions).

Figure 4. Size-effect exponent, $m$, as a function of Peierls stress, $\tau_{\mathrm{p}}$, for representative micropillars, indicates that when $\tau_{\mathrm{p}}<\sim 0.1 \mathrm{GPa}, m \approx 0.6-0.8$, showing a relatively consistent value; when $\sim 0.1 \mathrm{GPa}<\tau_{\mathrm{p}}<\sim 1 \mathrm{GPa}, 0<m<\sim 0.6-0.8$, showing $m$ decreases with increasing $\tau_{\mathrm{p}}$; when $\tau_{\mathrm{p}}>\sim 1 \mathrm{GPa}, m \approx 0$, showing almost no size effect; The values of $\tau_{\mathrm{p}}$ (or shear stress at $0 \mathrm{~K}$ for metallic glasses) are obtained from Refs. [44-48]. (It should be noted that Si, GaAs, quasicrystals (QCs) and metallic glasses (MGs) are all with the $m \approx 0$, the difference of their $m$ values is only for the legibility of the graph.) 


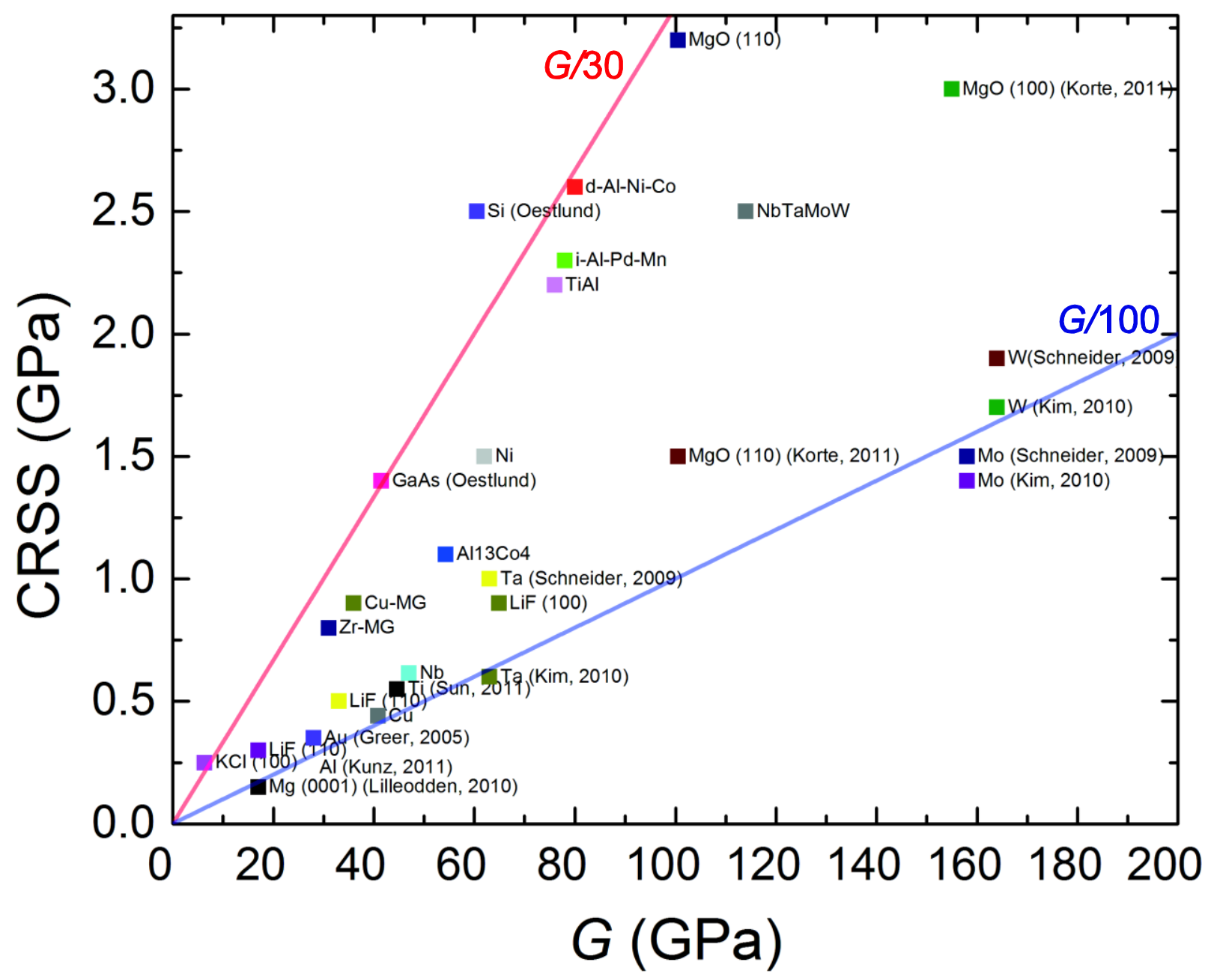




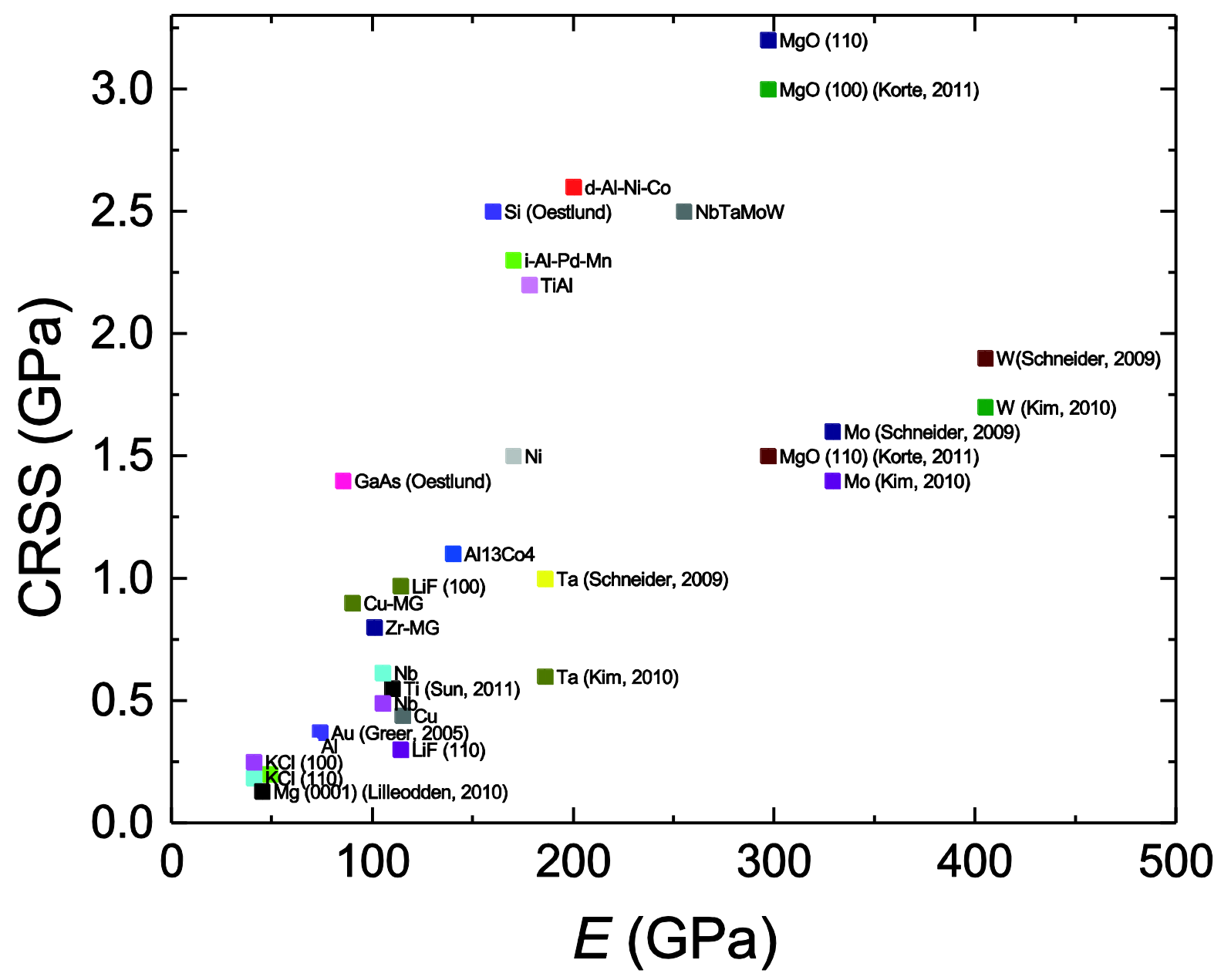




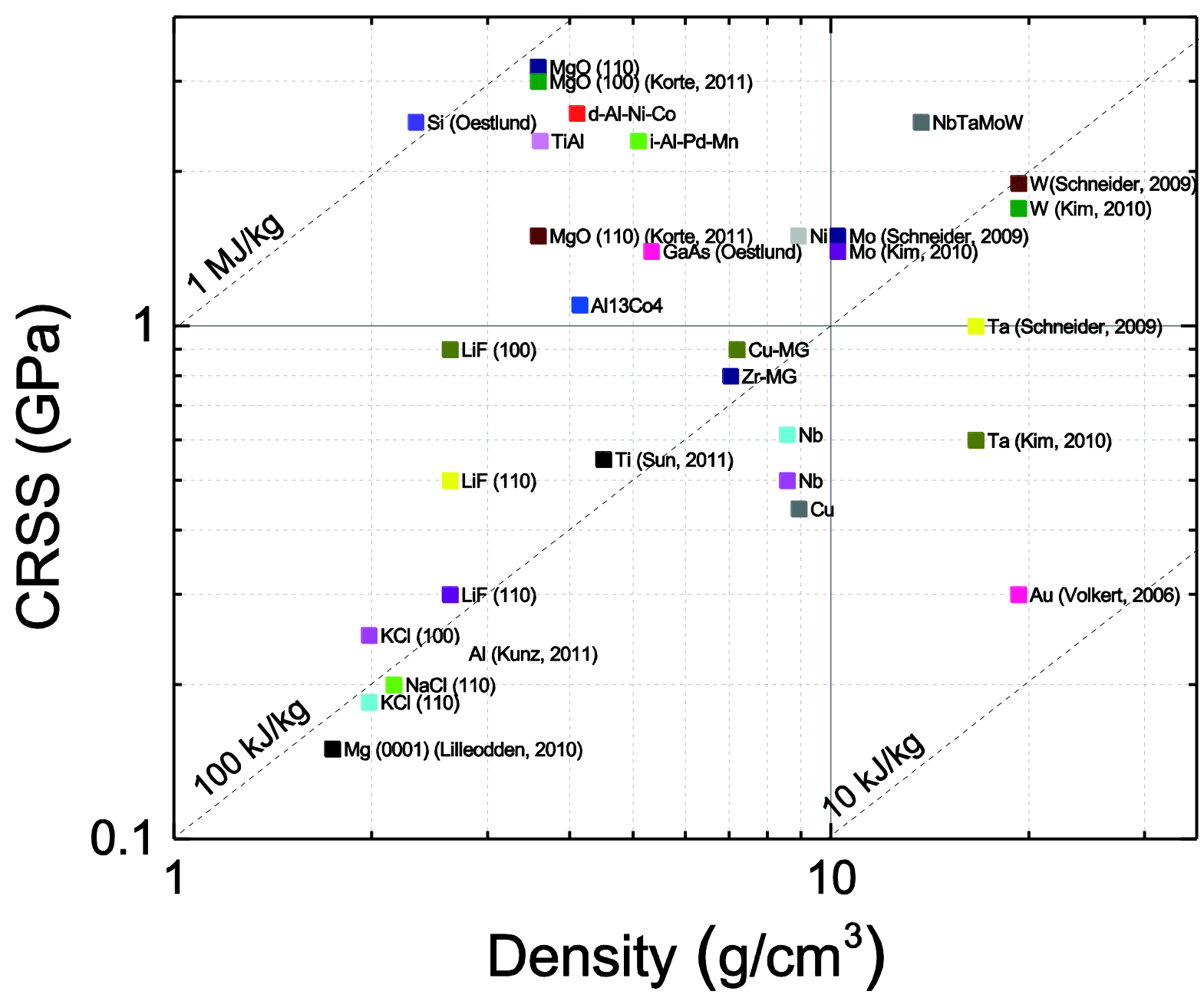




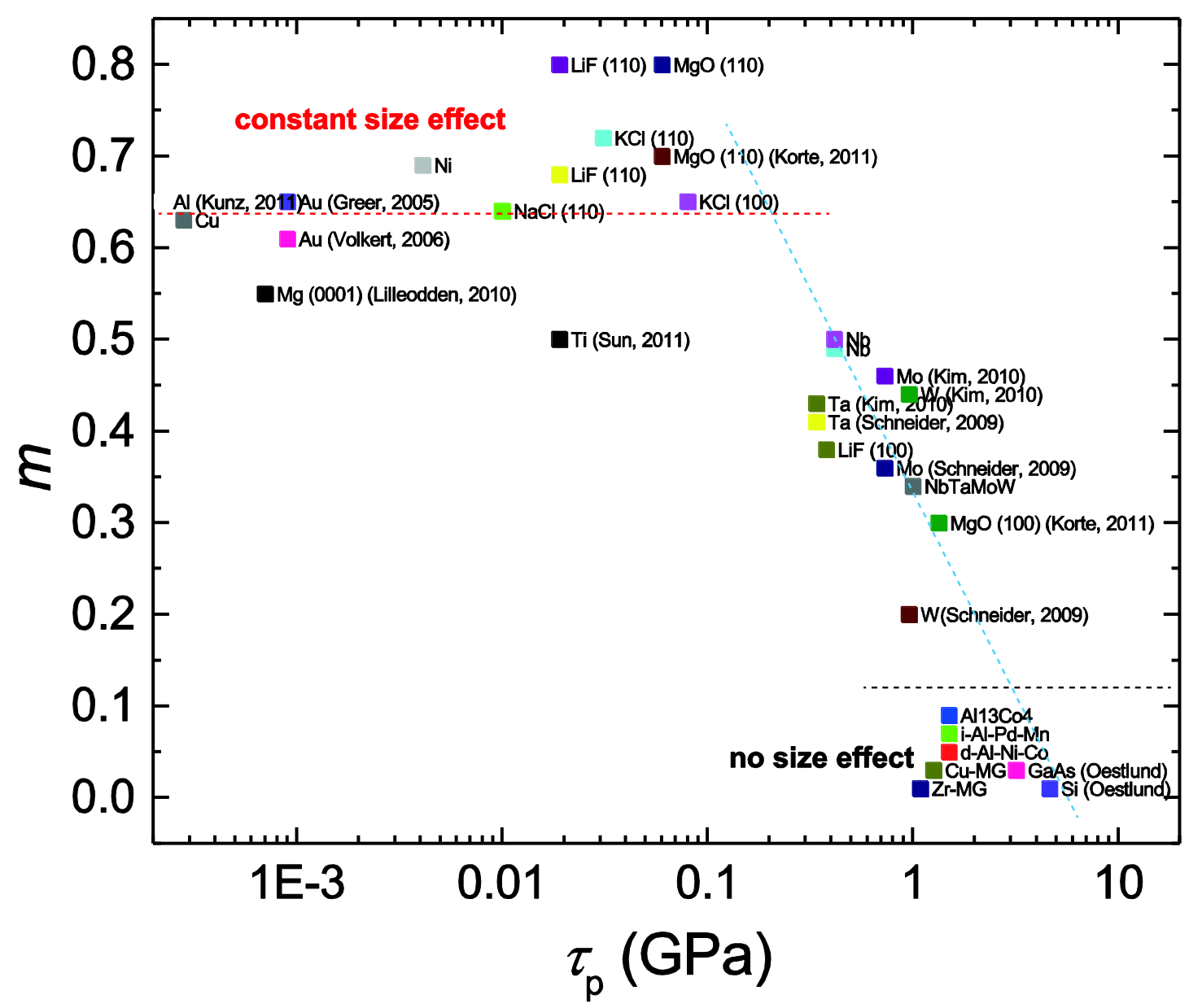


Table 1. Summary of representative materials on the compression of FIB-milled micro-/nano-pillars

\begin{tabular}{|c|c|c|c|c|c|c|c|c|c|c|c|c|c|c|c|}
\hline Materials & Au & Al & $\mathrm{Ni}$ & $\mathbf{C u}$ & Nb & Ta & Mo & W & V & Ti & Mg & $\mathrm{NaCl}$ & $\mathrm{KCl}$ & LiF & MgO \\
\hline Type & $\mathrm{fcc}$ & fcc & fcc & fcc & bcc & bcc & $\mathrm{bcc}$ & bcc & $\mathrm{bcc}$ & hcp & hcp & $\begin{array}{l}\text { ionic } \\
\text { crystal }\end{array}$ & $\begin{array}{l}\text { ionic } \\
\text { crystal }\end{array}$ & $\begin{array}{l}\text { ionic } \\
\text { crystal }\end{array}$ & $\begin{array}{l}\text { ionic } \\
\text { crystal }\end{array}$ \\
\hline Ref. & $\begin{array}{l}{[9,} \\
10]\end{array}$ & $\begin{array}{l}{[11,} \\
12]\end{array}$ & [13] & $\begin{array}{l}{[14,} \\
15]\end{array}$ & $\begin{array}{c}{[16,} \\
17]\end{array}$ & $\begin{array}{c}{[16,} \\
17]\end{array}$ & $\begin{array}{l}{[16,} \\
17]\end{array}$ & $\begin{array}{r}{[16,} \\
17]\end{array}$ & [36] & $\begin{array}{l}{[18,} \\
19]\end{array}$ & [20] & [21] & {$[22]$} & $\begin{array}{l}{[23,} \\
24]\end{array}$ & [25] \\
\hline Materials & \multicolumn{2}{|c|}{$\mathrm{Si}$} & GaAs & \multicolumn{2}{|c|}{ TiAl } & \multicolumn{2}{|c|}{ HEAs } & $\mathrm{Al}_{13} \mathrm{Co}_{4}$ & \multicolumn{2}{|c|}{ Al-Ni-Co } & \multicolumn{2}{|c|}{ Al-Pd-Mn } & $\begin{array}{c}\text { Cu-based } \\
\text { metallic } \\
\text { glass }\end{array}$ & \multicolumn{2}{|c|}{$\begin{array}{c}\text { Zn-based } \\
\text { metallic } \\
\text { glass }\end{array}$} \\
\hline Type & \multicolumn{2}{|c|}{$\begin{array}{l}\text { covalent } \\
\text { crystal }\end{array}$} & $\begin{array}{l}\text { covalent } \\
\text { crystal }\end{array}$ & \multicolumn{2}{|c|}{$\begin{array}{c}\text { ordered } \\
\text { intermetallic }\end{array}$} & \multicolumn{2}{|c|}{$\begin{array}{l}\text { disordered } \\
\text { intermetallic }\end{array}$} & $\begin{array}{c}\text { complex } \\
\text { metallic } \\
\text { alloy }\end{array}$ & \multicolumn{2}{|c|}{$\begin{array}{l}\text { decagonal } \\
\text { quasicrystal }\end{array}$} & \multicolumn{2}{|c|}{$\begin{array}{l}\text { icosahedral } \\
\text { quasicrystal }\end{array}$} & $\begin{array}{c}\text { metallic } \\
\text { glass }\end{array}$ & \multicolumn{2}{|c|}{$\begin{array}{l}\text { metallic } \\
\text { glass }\end{array}$} \\
\hline Ref. & \multicolumn{2}{|c|}{ [26] } & [27] & \multicolumn{2}{|c|}{ [28] } & \multicolumn{2}{|c|}{$[29,30]$} & [31] & \multicolumn{2}{|c|}{ [33] } & \multicolumn{2}{|c|}{ [32] } & [34] & \multicolumn{2}{|c|}{ [34] } \\
\hline
\end{tabular}

